\title{
Brain Endoscopy Under Local Anesthesia and Sedation: A Single-Center Experience of 510 Patients
}

\author{
Gurpreet S. Gandhoke ${ }^{1}$, BK Ojha ${ }^{2}$, Mazhar Husain ${ }^{3}$, Paolo Frassanito ${ }^{4}$, Peter Nakaji ${ }^{5}$ \\ 1. Department of Neurological Surgery, University of Pittsburgh Medical Center 2. Dept. of Neurosurgery, \\ King George's Medical University, Lucknow, India 3. Dept. of Neurosurgery, Sahara Hospital, Lucknow, \\ India 4. Dept. of Neurosurgery, Catholic University of Rome, Rome, Italy 5. Division of Neurological \\ Surgery, Barrow Neurological Institute
}

$\square$ Corresponding author: Gurpreet S. Gandhoke, gurpreet.gandhoke@gmail.com Disclosures can be found in Additional Information at the end of the article

\section{Abstract}

Objective: To report the results of endoscopic transcranial intervention with local anesthesia and sedation in a busy neurosurgical centre of India.

Methods: At the Department of Neurosurgery of the King George's Medical University India, more than 640 transcranial neuroendoscopic interventions have been performed for various indications over 11 years. All procedures were performed under sedation administered and monitored by a member of the neurosurgery team and local anesthesia. No head frame was used to affix the patient's head. Clinical charts from 510 patients were collected and retrospectively reviewed.

Results: Overall success rate for the endoscopy procedure was $75.1 \%$ (383 of 510 patients), failures being 127 (24.9\%). Fifty-four procedures had to be abandoned (10.6\%), and in the remaining 73 patients, the endoscopic treatment did not obtain the expected clinical result. Overall complication rate was $16.9 \%$ (86 patients) with a mortality rate of $1.2 \%$ (6 patients). Patients did not complain of any discomfort related to the sedation after the procedure, and we did not directly relate any surgical complication to the sedation.

Conclusions: This study proves the versatility and effectiveness of neuroendoscopy in the management of a wide group of neurosurgical conditions in a developing country. The feasibility of brain endoscopy under local anesthesia is also presented.

Received 12/10/2012 Review began 12/12/2012 Published 03/25/2013

\section{(C) Copyright 2013} Gandhoke et al. This is an open access article distributed under the terms of the Creative Commons Attribution License CC-BY 3.0., which permits unrestricted use, distribution, and reproduction in any medium, provided the original author and source are credited.
Categories: Neurosurgery

Keywords: local anesthesia, third ventriculostomy, biopsy, cyst excision, neuroendoscopy

\section{Introduction}

Neurosurgical centers of developing countries deal with a large number of patients despite meager economical resources. The health budget of India is $\$ 4.8$ billion for 1.2 billion people; by comparison the predicted health budget of United States for the year 2012 is $\$ 866$ billion. In this context, reducing the cost of treatment is pivotal to ensure health care to the largest portion of the population.

Neuroendoscopic surgery may prove very helpful to this purpose. Indeed, cost of endoscopic 
procedure is minor compared to open surgery and hospital stay is reduced, resulting in further saving of economical resources [1]. Finally, the avoidance of shunt placement leads to a reduction in complications for the patients [2], requiring fewer hospitalizations and emergent procedures.

Furthermore, the burr hole approach to neuroendoscopy makes it amenable to local anesthesia and sedation, although general anesthesia is preferable to ensure immobility [3]. Given the relative scarcity of the availability of an anesthesiologist in emergency settings, commonly encountered in developing countries, we chose to perform endoscopic procedures under local anesthesia combined with sedation.

In this paper, we report our 11-year experience in the use of neuroendoscopy under local anesthesia and sedation in the treatment of several pathological conditions. Technical difficulties encountered by the operating surgeon, their solutions, as well as complications and their management are detailed.

\section{Materials And Methods}

The records of 510 of the 640 patients who underwent transcranial endoscopic interventions at the Department of Neurosurgery at the King George's Medical University, India, from March 1996 to December 2007, were collected and retrospectively reviewed. Age, gender, clinical presentation, type of endoscopic procedure, and outcome were analyzed.

Age ranged from 15 days to 84 years (mean 20.21 years). There were 370 males and 140 females (male/female ratio $=2.6$ ). We treated 299 patients younger than 18 years old (Pediatric group) and 211 patients older (Adult group).

\section{Outcome and definition of success}

The goals of endoscopic surgery were classified as follows: to restore cerebrospinal fluid (CSF) flow, to fenestrate cyst walls or intraventricular septations, to drain the cyst content, and to excise a lesion or perform a biopsy, or finally any combination of the above-mentioned.

In the postoperative period, lumbar puncture was routinely performed after third ventriculostomy (TV) for three consecutive days, if not contraindicated by the presence of a posterior fossa mass.

The clinical outcome was limited to the findings obtained during the same hospital stay in which the endoscopic procedure was performed. Clinical and radiological criteria were evaluated in order to state the achievement of the goal defined for a particular procedure. The retrospective nature and the short follow-up restrained us from any neuropsychological assessment.

Success was defined as no need of further surgery to achieve the goal defined for the endoscopic procedure. If patient's required surgical excision based upon their histopathology after endoscopic biopsy, this procedure was nonetheless considered successful.

On the other hand, procedures that were abandoned because of intraoperative complications or technical difficulties and absence of any neurological improvement after a completed procedure, requiring further intervention, were considered failures.

\section{Instrumentation}


The Gaab universal endoscope (Karl-Storz, Tuttlingen, Germany) was used. The set is constituted by a working sheath (6.5 mm outer diameter, working channels $1 \mathrm{~mm}$ and $2.7 \mathrm{~mm})$ and 0 -degree or 30-degree telescopes ( $2.7 \mathrm{~mm}$ and $4 \mathrm{~mm}$ diameter, respectively). The neurofiberscope ( $2.5 \mathrm{~mm}$, Karl Storz, Tuttlingen, Germany) was used in procedures involving the posterior fossa (inspection of the aqueduct and fourth ventricle, aqueductoplasty, biopsy of posterior fossa tumors). A television monitor and a single-chip camera were attached to the endoscope for visual control and teaching.

\section{Procedure: general principles}

The patient is placed in the supine position with the head slightly flexed and fixed with an adhesive tape across the table. Sedation, typically using Ketamine (1-2 mg/Kg IV) and a mixture of Pentazocin and Phenergan was utilized. Local anesthesia (lignocaine $2 \%$ diluted $50 \%$ with normal saline) was administered at the incision site by the resident.

A burr hole was placed based on information obtained from computerized tomography (CT) or magnetic resonance (MR) imaging. After the dura was opened, the working sheath containing the trocar was inserted with a freehand technique and fixed by the endoscope holder (Aesculap, Tuttlingen, Germany). The trocar was removed and the rigid scope was introduced.

Continuous irrigation with Ringer's lactate solution was used to maintain a clear view. Warm Ringer's lactate solution was used for irrigation whenever bleeding occurred. Dangerous increases in intracranial pressure (ICP) were avoided by maintaining a permissible inflow (about $10 \mathrm{ml} / \mathrm{min}$ ) with sufficient outflow of irrigating fluid. Minor bleeding usually stopped after a few minutes of irrigation. Copious irrigation or suction was used to remove clots.

When the procedure was completed, the operating sheath and the endoscope were withdrawn in one piece to look for active bleeding along the cortical trajectory. Gel foam sponge was used to pack the burr hole. The galea and skin were closed with interrupted sutures. Stitches were removed on the sixth to eighth postoperative day.

\section{Procedure: particular technical aspects}

Endoscopic third ventriculostomy (ETV) was performed anterior to the mammillary body in the region of the hypophyseal fossa. A 1-mm (No. 3 French) Fogarty catheter was used either to perforate the floor of the third ventricle or to dilate the stoma.

Septostomy or cysto-ventriculostomy was performed using a bipolar electrosurgical probe. Additionally, a Fogarty catheter was used to dilate the stoma.

Cystic lesions were opened and the cystic wall was shrunk by means of an electrosurgical probe. The cyst content was aspirated using an angiographic catheter.

Ventricular tumors were inspected in order to identify the feeding arteries, 30 and 70 degree telescopes being useful in visualizing the tumors located in the posterior portion of the third ventricle. Biopsy and eventual excision of the mass were performed by suction using an angiographic catheter.

\section{Results}

We performed 784 endoscopic gestures in 510 procedures (Table 1). TV was the most common, being performed in 402 cases, as either an unique treatment for hydrocephalus or concomitantly with other endoscopic gestures. 


\section{Cureus}

\section{Procedure}

Third ventriculostomy

Biopsy

Septostomy

Excision of lesion

Monroplasty

Aqueductoplasty

Cystoventriculostomy

Tumor decompression

Cystocisternostomy

Shunt retrieval

Choroid plexus fulguration

Fenestration of entrapped temporal horn

Aqueductal stent placement

Total

\section{Number of Cases}

402

116

112

52

26

21

20

19

8

4

2

2

1

784

TABLE 1: Summary of the endoscopic procedures performed either singularly or concomitantly in a total of $\mathbf{5 1 0}$ procedures.

The endoscopic procedure was abandoned in 54 cases (10.6\%). The main causes are detailed in Table 2. 


\section{Cureus}

\section{Condition}

Abnormal anatomy +/- turbid CSF*

Bleeding hampering vision**

Technical faults ${ }^{\star \star \star}$

Extreme macrocephaly ${ }^{\star \star \star *}$

Overall rate of abandoned procedures

\section{Number of Cases}

34

15

30

2

$10.6 \%(54 / 510)$

\section{TABLE 2: Summary of conditions contributing to abandonment of the endoscopic procedure attempt.}

*Including non-visualization of the foramen of Monro, absent septum pellucidum, cicatrisation of the foramen of Monro. Orientation was further complicated in post-infectious hydrocephalus by the presence of hazy CSF. ** Bleeding not controlled with usual techniques, like irrigation with warm Ringer's lactate, especially encountered in patients with a very large head and very friable ependymal walls of the lateral ventricular system. ${ }^{* \star *}$ Including inadvertent opening of a large area of the dura resulting in leakage of the irrigating fluid ( 2 cases) and inability to visualize the ventricular system (1 case). ${ }^{\star \star \star \star}$ Requiring longer scopes to reach area of interest.

The overall success rate was $75.1 \%$ (383 out of 510 cases), without significant difference between the Adult and the Pediatric Group (73.9\% and $75.9 \%$, respectively). Table 3 details the success rates of endoscopic surgery per diagnosis in the pediatric and adult populations.

Three hundred and fifty-seven patients with successful treatment and without postoperative complication had a short mean hospital stay ( $3 \pm 1.4$ days). The remaining 153 patients experiencing any complication and/or failure of the endoscopic treatment, thus requiring intermittent lumbar drainage for CSF leakage, shunts, microsurgery, and/or repeat endoscopic surgery, had a longer mean hospital stay (9.2 \pm 4.3 days).

\begin{tabular}{|c|c|c|c|c|c|}
\hline \multirow{2}{*}{$\begin{array}{l}\text { Diagnosis } \\
\text { (No. pts) }\end{array}$} & \multirow{2}{*}{$\begin{array}{l}\text { Surgical Goal } \\
\text { Restoration of CSF }\end{array}$} & \multicolumn{2}{|l|}{ Adult } & \multicolumn{2}{|l|}{ Prediatric } \\
\hline & & Success & Failure & Success & Failure \\
\hline & Flow $=1$ & No. (\%) & $\begin{array}{l}\text { No. } \\
(\%)\end{array}$ & No. (\%) & $\begin{array}{l}\text { No. } \\
\text { (\%) }\end{array}$ \\
\hline & Fenestration = 2 & & & & \\
\hline & Drainage of cyst & & & & \\
\hline & $\begin{array}{l}\text { Content/intracventricular } \\
\text { clot }=3\end{array}$ & & & & \\
\hline & Excision/biopsy = 4 & & & & \\
\hline $\begin{array}{l}\text { Tubercular Meningitis with } \\
\text { Hydrocephalus (104) }\end{array}$ & $1-4$ & $24(77)$ & $7(23)$ & 66(90) & $7(10)$ \\
\hline Multiloculated Hydrocephalus (30) & $1-2$ & 0 & 0 & 18(60) & $12(40)$ \\
\hline
\end{tabular}




\section{Cureus}

Infectious

Post-Meningitis Aqueductal Stenosis (33)

1

Neurocysticercosis (29)

$1-4$

Medullablastoma (39)

Midline Cerebellar Astrocytoma (26)

$1-4$

Ependymoma (3)

Acoustic Tumors (8)

Tumor Pineal Region Tumors (27)

Craniopharyngioma (11)

Thalamic Glioma (11)

Brainstem Glioma (10)

Tectal Glioma (3)

Colloid Cyst (20)

Arachnoid Cyst (12)

Ependymal Cyst (9)

Cyst

Others

Suprasellar Arachnoid Cysts (4)

Septum Pellucidum Cyst (3)

Choroid Plexus Cyst (2)

Aqueductal Stenosis (77)

Dandy-Walker (16)

Hydrocephalus Associated with

Spinal Dysraphism (13)

Post-Traumatic Hydrocephalus (9) 1 - 3

Communicating Hydrocephalus(9) 1

Virchov-Robin Spaces Dilation with

Hydrocephalus (1)

Basilar Tip Aneurysm with

Hydrocephalus (1)

Overall Success Rate
$1-4$

1

$1-4$

$1-3-4$

$1-4$

$1-4$

$1-4$

$1-3-4$

$1-2$

$1-2-4$

$1-2$

$1-2$

$1-2-4$

1

1

1

1

1

Total

$75.1 \%$
$8(50) \quad 8(50) \quad 11(65) \quad 6(35)$

$18(95) \quad 1(0.05) \quad 9(90) \quad 1(0.1)$

$3(75) \quad 1(25) \quad 21(60) \quad 14(40)$

$15(65) \quad 8(35) \quad 2(67) \quad 1(33)$

$2(67) \quad 1(33) \quad 0 \quad 0$

$7(88) \quad 1(12) \quad 0 \quad 0$

$10(56) \quad 8(44) \quad 6(67) \quad 3(33)$

$1(33) \quad 2(67) \quad 3(38) \quad 5(63)$

$8(73) \quad 3(27) \quad 0 \quad 0$

$1(50) \quad 1(50) \quad 5(63) \quad 3(37)$

$\begin{array}{llll}0 & 0 & 2(67) & 1(33)\end{array}$

$15(83) \quad 3(17) \quad 1(50) \quad 1(50)$

$8(89) \quad 1(11) \quad 3(100) \quad 0$

$4(80) \quad 1(20) \quad 4(100) \quad 0$

$3(75) \quad 1(25) \quad 0 \quad 0$

$\begin{array}{llll}0 & 0 & 3(100) & 0\end{array}$

$1(100) \quad 0 \quad 1(100) \quad 0$

$13(76) \quad 4(24) \quad 54(90) \quad 6(10)$

$\begin{array}{llll}0 & 1(100) & 7(47) & 8(53)\end{array}$

$1(50) \quad 1(50) \quad 8(73) \quad 3(27)$

7 (78) $\quad 2(22) \quad 0 \quad 0$

$5(100) \quad 0 \quad 3(75) \quad 1(25)$

$1(100) \quad 0 \quad 0 \quad 0$

$1(100) \quad 0 \quad 0 \quad 0$

Adult Pedatric

$73.9 \% \quad 75.9 \%$

TABLE 3: Success rate of endoscopy detailed per pathology 
Infectious: Endoscopic procedure was successful in 90 patients (out of $104=86.5 \%$ ) with tuberculous meningitis hydrocephalus (TBMH), in 18 patients (out of $30=60 \%$ ) with loculated hydrocephalus, in 19 (out of $33=57.6 \%$ ) with post-meningitis aqueductal stenosis, and in 27 (out of $29=93.1 \%$ ) affected by neurocysticercosis (NCC).

In case of acquired aqueductal stenosis and multiloculated hydrocephalus, the goal of endoscopy was exclusively to restore the CSF circulation. In TBMH and NCC, endoscopy carried additional advantages. Indeed, in seven patients, the endoscopic treatment allowed to pose the diagnosis of TBMH, since multiple tiny lesions on the wall of the right lateral and third ventricles were visualized. Pathological findings were consistent with tuberculous granulomas. These lesions were not visible on imaging. In patients affected by NCC, the endoscopic extirpation of the third and fourth ventricular NCC cyst was successful in $81.3 \%(n=16)$ and $92 \%$ $(\mathrm{n}=13)$ of the cases, respectively.

Tumors: 138 patients affected by tumor were treated endoscopically, with a success rate of 62.3\%. Main goal of the endoscopic treatment was the restoration of CSF flow by means of TV, with or without septostomy.

In patients harboring a tumor extending in the ventricular system, endoscopic biopsy was undertaken concomitantly with the treatment of hydrocephalus.

Patients with predominantly cystic craniopharyngiomas were subjected to endoscopic resection, with gross total excision achieved in 9 out 11 cases (82\%). Two patients died because of severe hemorrhage secondary to an intraoperative traumatic injury of the basilar artery in one and the exact source of bleeding could not be pointed out in the other.

Cysts: In the group of patients affected by cystic non-neoplastic lesions, the success rate of endoscopic treatment was largely higher (86\%) if compared to neoplastic lesions. The pathology with the lower chance of success was the colloid cyst (75\%). Indeed, one patient required microsurgical excision of the residual cyst, which had a very thick wall and could not be removed endoscopically; two patients underwent re-do endoscopic treatment, since the postoperative radiological image showed a significant residual cyst wall associated with persistent hydrocephalus. Two patients died from meningitis, unrelated to the endoscopic procedure.

Interestingly, one third ventricular ependymal cyst was diagnosed intraoperatively as isointense to CSF on MRI and preoperatively misdiagnosed as aqueductal stenosis.

Others: Seventy-seven patients affected by primary aqueductal stenosis were treated with a success rate of $87 \%$, significantly higher than the acquired form (57.6\%).

Finally, the endoscopic treatment treated hydrocephalus in seven patients with Dandy-Walker malformation (out of $16,43.8 \%$ ), nine cases with dysraphism (out of 13, 61.5\%), seven cases with communicating hydrocephalus (out of nine, $78 \%$ ), eight cases with previous traumatic brain injury (out of nine, 89\%), one patient with Virchow-Robin spaces dilation, and one patient with basilar tip aneurysm.

\section{Complications and their management}

We did not observe any surgical complication directly related to the sedation. 


\section{Cureus}

Complications of endoscopic procedures are detailed in Table 4. The overall complication rate was $16.9 \%$ (86 out of 510 patients). Mortality was $1.2 \%$ (six patients).

\begin{tabular}{lc}
\hline Complication & Number \\
\hline CSF leak & $43(8.4)$ \\
\hline Bleeding & $34(6.7)$ \\
\hline Wound Infection & $12(2.4)$ \\
\hline Meningitis & $11(2.2)$ \\
\hline Subdural hematoma & $3(0.6)$ \\
\hline Paresis of cranial nerves III and VI & $3(0.6)$ \\
Hypothalamic dysfunction & $2(0.4)$ \\
Hemiparesis & $1(0.2)$ \\
Mortality & $6(1.2)$ \\
Overall complication rate
\end{tabular}

\section{TABLE 4: Summary of complications of neuroendoscopic procedures.}

* Bleeding led to death in two patients with a solid craniopharyngioma and in one patient with multiloculated hydrocephalus. ** Meningitis led to death in two patients with colloid cysts and in one patient with multiloculated hydrocephalus. ${ }^{\star \star \star}$ Expressing the number of patients experiencing one or more perioperative complications.

Bleeding: Two patients with a colloid cyst experienced major bleeding when the surgeon attempted to separate the wall from the septal vein. The bleeding was controlled endoscopically in one patient. However, the procedure was abandoned and the patient underwent microsurgical resection of the cyst two weeks later. The other patient required placement of an external ventricular drain for five days. Two patients with multiloculated hydrocephalus experienced major bleeding, leading to exitus in one of them. Two patients treated for a craniopharyngioma died due to severe hemorrhage during an attempt to coagulate the tumor wall (see results). The source of bleeding was identified as the basilar artery in one case but could not be identified in the other case.

CSF leak: In 43 patients affected by hydrocephalus, a CSF leak from the scalp incision site was observed before or at the time of stitch removal. In 13 patients, this complication was successfully managed by intermittent lumbar drainage of CSF for three to five days, along with oral acetazolamide for two to three weeks. Thirty patients finally required a CSF shunt placement.

Meningitis: The postoperative course was complicated by meningitis in 11 patients, requiring external ventricular drainage to control the hydrocephalus. Nonetheless, this complication was fatal in two patients with colloid cysts and in one patient with multiloculated hydrocephalus.

Others: Three patients required the surgical evacuation of a subdural hematoma. 
Postoperative neurological deficit was observed in four cases, three cases showing cranial nerve impairment after transaqueductal access to the fourth ventricle or while performing aqueductoplasty and the remaining one hemiparesis.

Finally, wound infection constituted a minor complication, usually managed conservatively.

\section{Discussion}

Minimal invasiveness, wide visualization of anatomy, negligible brain retraction and blood loss, reduced surgical manipulation and trauma, and milder postoperative reactions are wellestablished benefits of neuroendoscopy. Moreover, the versatility of endoscopy allows to concomitantly treat CSF flow obstruction, and perform biopsy or remove a tumor in a single stage procedure. All these aspects are reflected by decreased expense in terms of required material if compared to open surgery and shortened hospitalizations, further resulting in reduction of cost for the health care system. All these advantages are confirmed by our large experience.

Moreover, at our institution all procedures were performed by means of a single neuroendoscopy set, regular maintenance being undertaken by the neurosurgical staff for the entire period of study. This scenario carried further economical advantage, since the cost of disposable items and constant replacement of endoscopic sets makes them prohibitively expensive. In a developing country, this reduction of cost of health care is pivotal in order to ensure a more standard treatment and to make it accessible to almost everyone, as demonstrated by the experience of Warf in a referral mission hospital in Uganda [4] and by the promising results of a mobile neuroendoscopy unit [5]. We would like to highlight that we performed all the procedures collected in this report under local anesthesia and sedation, since an anesthesiologist is not readily available to assist all the emergency procedures, given the number of patients treated daily in our institution.

Although general anesthesia is preferable to ensure immobility, our anesthesiological management never restrained us from accomplishment of the procedure. Indeed, the procedure was abandoned in $10.6 \%$ of cases (54/510 patients), in almost two-thirds of the cases (34 patients) as a consequence of the intraoperative evidence of an abnormal anatomy. This is partially justified by the lack of high-resolution radiologic study in the emergency setting in our institution. Nonetheless, no major complications nor patient discomfort related to the use of sedation and local anesthesia was observed. Cardiovascular instability has been reported during neuroendoscopic procedures, occurring in $28-32 \%$ of patients, but these events are selflimiting, as we also found, and rapidly improve with release of ICP [3].

The overall success rate was $75.1 \%$ (383/510 patients). Although this is hard to compare this data with current literature, as most of the papers focus on the outcome of a specific procedure, as for example TV rather than septostomy, the effectiveness of endoscopy in particular conditions was undoubted. Indeed, endoscopy allowed us to avoid the placement of a shunt in $73.5 \%$ of patients with hydrocephalus (319 of 434). In our opinion, endoscopy should be the ideal first step of treatment in pediatric patients affected by hydrocephalus, as benefit of avoiding shunt as well as its maintenance and complications are more prominent due to their longer life expectance [6].

It is also of worth to note the prevalent role that endoscopy is assuming to treat pathologies endemic in developing countries, such as tubercular meningitis. In the present series, the successful rate in TBM-related hydrocephalus was as high as $85.1 \%$. Although this result is limited by the absence of follow-up in the present study, in a previous report the successful rate of endoscopic TV in our institution was 73.1\% [7]; a review of the literature has disclosed a rate of $65-68 \%$ [8], thus confirming the particular advantage of endoscopy in such a type of 
hydrocephalus as shunting is burdened by a high risk of complication [9].

Furthermore, endoscopy can also play an important role as a diagnostic tool, as high-detail radiological examinations are not available. Although tubercular meningitis is usually diagnosed on the basis of CSF examination and imaging features, demonstration of mycobacterium tuberculosis in smear or on culture is possible in only a small number of patients [10]. In our experience, 10 patients affected by hydrocephalus without clinical or imaging features suggestive of tuberculous infection were diagnosed based on the demonstration of granulomas in tubercles biopsied while undergoing endoscopic TV, thus allowing immediate institution of anti-tuberculous therapy.

Similarly, in a patient with a fourth ventricular ependymoma, endoscopy showed third ventricular seeding that was not evident on imaging studies. This information helped us to stage the neoplastic disease and tailor the planning of radiotherapy treatment.

Our overall complication rate was $16.9 \%$ (86/510 patients) with a mortality of $1.2 \%(6 / 510$ patients). Although series from developed countries reported complications rate inferior to $15 \%$ [11-13], we think that the different etiology, namely, the prevalence of post-infectious hydrocephalus, could account for the increased rate of complications. Indeed, a recent analysis suggested that endoscopy outcome in developing countries should be adjusted for the etiology in order to be comparable with developed countries [14]. Moreover, some patients are severely compromised and malnourished, this factor also affecting the risk of postoperative complications.

We observed a mortality rate of $1.2 \%$ (6/510 patients), only slightly higher than the mortality rate (less than 1\%) reported in the literature $[13,15]$. To attribute this either to the fact that we did not have general anesthesia during these surgeries or to the fact that we had to accomplish all that we could surgically in the same setting is difficult to answer. Indeed, two patients died following an intraoperative hemorrhage complicating an attempt to dissect a craniopharyngioma away from the basilar artery. Therefore, we recommend not performing complex endoscopic procedures, such as the removal of craniopharingioma, without adequate anesthesiological assistance. If emergency treatment is required, an endoscopic procedure aimed to relieve the intracranial pressure and/or the mass effect of the tumor on neural structures is performed, thus allowing planning a second-step elective surgery under general anesthesia. As of January 2008, we have stopped performing endoscopic interventions under local anesthesia. This study underlines the feasibility of achieving the goal to save a life in the most trying circumstances with the best that is available.

\section{Conclusions}

Neuroendoscopy is effective in the treatment of several hydrocephalic conditions and can also be useful as a diagnostic tool in developing countries. A crucial advantage of endoscopic treatment is to shorten hospital stays with a consequent reduction in costs and a rapid turnover of patients.

Although we do not advocate quitting the use of general anesthesia in neuroendoscopy, our experience affirms that brain endoscopy is also feasible with local anesthesia and sedation without significant increase in morbidity or discomfort for the patient. This evidence is certainly important to propel the widespread use of endoscopy in developing countries.

\section{Additional Information}

\section{Disclosures}


Human subjects: Consent was obtained by all participants in this study. The IRB committee of the King George's Medical University, India issued approval N/A. Animal subjects: All authors have confirmed that this study did not involve animal subjects or tissue. Conflicts of interest: In compliance with the ICMJE uniform disclosure form, all authors declare the following:

Payment/services info: All authors have declared that no financial support was received from any organization for the submitted work. Financial relationships: All authors have declared that they have no financial relationships at present or within the previous three years with any organizations that might have an interest in the submitted work. Other relationships: All authors have declared that there are no other relationships or activities that could appear to have influenced the submitted work.

\section{References}

1. Warf BC: Hydrocephalus in Uganda: The predominance of infectious origin and primary management with endoscopic third ventriculostomy. J Neurosurg. 2005, 102:1-15.

2. Di Rocco C, Massimi L, Tamburrini G: Shunts vs endoscopic third ventriculostomy in infants: are there different types and/or rates of complications? A review. Childs Nerv Syst. 2006, 22:1573-1589.

3. Schubert A, Deogaonkar A, Lotto M, Niezgoda J, Luciano M: Anesthesia for minimally invasive cranial and spinal surgery. J Neurosurg Anesthesiol. 2006, 18:47-56.

4. Warf BC: Comparison of endoscopic third ventriculostomy alone and combined with choroid plexus cauterization in infants younger than 1 year of age: A prospective study in 550 African children. J Neurosurg. 2005, 103:475-481.

5. Qureshi MM, Piquer J, Young PH: Mobile endoscopy: a treatment and training model for childhood hydrocephalus. World Neurosurg. 2013, 79(2 Suppl):S24:e1-4. 10.1016/j.wneu.2012.02.001

6. Hopf NJ, Grunert P, Fries G, Resch KD, Perneczky A: Endoscopic third ventriculostomy: Outcome analysis of 100 consecutive procedures. Neurosurgery. 1999, 44:795-804.

7. Chugh A, Husain M, Gupta RK, Ojha BK, Chandra A, Rastogi M: Surgical outcome of tuberculous meningitis hydrocephalus treated by endoscopic third ventriculostomy: prognostic factors and postoperative neuroimaging for functional assessment of ventriculostomy. J Neurosurg Pediatr. 2009, 3:371-377.

8. Bhagwati S, Mehta N, Shah S: Use of endoscopic third ventriculostomy in hydrocephalus of tubercular origin. Childs Nerv Syst. 2010, 26:1675-1682.

9. Smyth MD, Tubbs RS, Wellons JC, 3rd, Oakes WJ, Blount JP, Grabb PA: Endoscopic third ventriculostomy for hydrocephalus secondary to central nervous system infection or intraventricular hemorrhage in children. Pediatr Neurosurg. 2003, 39:258-263.

10. Tiel R, Rosenblum, M.L: Chronic granulomatous lesions: tuberculosis, leprosy, sarcoidosis . Neurosurgery. Wilkins RH, Rengachary, S.S. (ed): McGraw-Hill, New York; 1996. 3341-3350.

11. Cinalli G, Spennato P, Ruggiero C, Aliberti F, Trischitta V, Buonocore MC, et al.: Complications following endoscopic intracranial procedures in children. Childs Nerv Syst. 2007, 23:633-644.

12. Peretta P, Ragazzi P, Galarza M, Genitori L, Giordano F, Mussa F, et al.: Complications and pitfalls of neuroendoscopic surgery in children. J Neurosurg. 2006, 105:187-193.

13. Schroeder HW, Oertel J, Gaab MR: Incidence of complications in neuroendoscopic surgery . Childs Nerv Syst. 2004, 20:878-883.

14. Kulkarni AV, Warf BC, Drake JM, Mallucci CL, Sgouros S, Constantini S: Surgery for hydrocephalus in sub-Saharan Africa versus developed nations: a risk-adjusted comparison of outcome. Childs Nerv Syst. 2010, 26:1711-1717.

15. Beems T, Grotenhuis JA: Long-term complications and definition of failure of neuroendoscopic procedures. Childs Nerv Syst. 2004, 20:868-877. 